\title{
Fibroblast Growth Factor Receptor Signaling Promotes Radial Glial Identity and Interacts with Notch1 Signaling in Telencephalic Progenitors
}

\author{
Keejung Yoon, ${ }^{1,2}$ Susana Nery, ${ }^{4 \star}$ Michael L. Rutlin, ${ }^{4}$ Freddy Radtke, ${ }^{5}$ Gord Fishell, ${ }^{4}$ and Nicholas Gaiano ${ }^{1,2,3,4}$ \\ ${ }^{1}$ Institute for Cell Engineering, Departments of ${ }^{2}$ Neurology and ${ }^{3}$ Neuroscience, Johns Hopkins University School of Medicine, Baltimore, Maryland 21205, \\ ${ }^{4}$ Developmental Genetics Program and Department of Cell Biology, Skirball Institute of Biomolecular Medicine, New York University School of Medicine, \\ New York, New York 10016, and ${ }^{5}$ Ludwig Institute for Cancer Research, Chemin de Boveresses 155, CH-1066 Epalinges, Switzerland
}

\begin{abstract}
The Notch and fibroblast growth factor (FGF) pathways both regulate cell fate specification during mammalian neural development. We have shown previously that Notch1 activation in the murine forebrain promotes radial glial identity. This result, together with recent evidence that radial glia can be progenitors, suggested that Notch1 signaling might promote progenitor and radial glial character simultaneously. Consistent with this idea, we found that in addition to promoting radial glial character in vivo, activated Notch1 (ActN1) increased the frequency of embryonic day 14.5 (E14.5) ganglionic eminence (GE) progenitors that grew into neurospheres in FGF2. Constitutive activation of C-promoter binding factor (CBF1), a Notch pathway effector, also increased neurosphere frequency in FGF2, suggesting that the effect of Notch1 on FGF responsiveness is mediated by CBF1.

The observation that ActN1 promoted FGF responsiveness in telencephalic progenitors prompted us to examine the effect of FGF pathway activation in vivo. We focused on FGFR2 because it is expressed in radial glia in the GEs where ActN1 increases FGF2 neurosphere frequency, but not in the septum where it does not. Like ActN1, activated FGFR2 (ActFGFR2) promoted radial glial character in vivo. However, unlike ActN1, ActFGFR2 did not enhance neurosphere frequency at E14.5. Additional analysis demonstrated that, unexpectedly, neither ActFGFR2 nor ActFGFR1 could replace the need for ligand in promoting neurosphere proliferation. This study suggests that telencephalic progenitors with radial glial morphology are maintained by interactions between the Notch and FGF pathways, and that the mechanisms by which FGF signaling promotes radial glial character in vivo and progenitor proliferation in vitro can be uncoupled.
\end{abstract}

Key words: Notch; FGF; CBF1; radial glia; telencephalon; progenitor

\section{Introduction}

The timing with which neural progenitors produce postmitotic cells during brain development has been well characterized. However, less is known about the molecular regulation of this process, in particular with respect to progenitor maintenance, proliferation, and heterogeneity. We have focused on the role of Notch and fibroblast growth factor (FGF) signaling in the neural progenitor pool of the developing mouse forebrain. In the vertebrate nervous system, the Notch pathway was traditionally

Received March 17, 2004; revised Sept. 12, 2004; accepted Sept. 13, 2004.

This work was supported by a postdoctoral fellowship from the American Cancer Society (PF4473 to N.G.), a Career Award in the Biomedical Sciences from the Burroughs Wellcome Fund (N.G.), a grant from the Children's Brain Tumor Foundation (G.F.), and a grant from the National Institute of Neurological Disorders and Stroke (NS32993 to G.F.). Additional funding was provided by PRAXIS XXI through the Gulbenkian Ph.D. program (S.N.). We thank John Hirst and Karen Chadwick for cell sorting, Jean Hebert and Sue McConnell for the foxg1::CRE mice, and Muriel Cleary and Yuanyuan Huang for technical assistance. We also thank Michelle Starz-Gaiano and Alex Joyner for critical reading of this manuscript. In addition, we thank Steve Burden, Alka Mansukhani, and Moosa Mohammadi for the activated ErbB2, FGFR2, and FGFR1 cDNAs, respectively.

${ }^{*}$ K.Y. and S.N. contributed equally to this work.

Correspondence should be addressed to either of the following: Gord Fishell at the above address, E-mail: fishell@saturn.med.nyu.edu; or Dr. Nicholas Gaiano, Johns Hopkins University School of Medicine, Institute for Cell Engineering, 733 North Broadway, BRB 715, Baltimore, MD 21205, E-mail: gaiano@jhmi.edu.

DOI:10.1523/JNEUROSCI.0993-04.2004

Copyright $\odot 2004$ Society for Neuroscience $\quad$ 0270-6474/04/249497-10\$15.00/0 thought to maintain a progenitor state while inhibiting differentiation (Lewis, 1996). Gain-of-function studies in the frog and chick have found that activation of the Notch pathway prevents progenitors from undergoing neurogenesis, whereas blocking this pathway leads to excessive neurogenesis and depletion of the progenitor pool (Chitnis, 1995; Henrique et al., 1997). Similarly, in mammals, widespread Notch activation expands the neural progenitor pool at the expense of neuron production (Lardelli et al., 1996), whereas disruption of Notch signaling leads to precocious expression of early neuronal markers (de la Pompa et al., 1997).

More recently, gain-of-function studies have found that Notch signaling not only inhibits the differentiation of some cell types but can also promote glial fate (Furukawa et al., 2000; Gaiano et al., 2000; Morrison et al., 2000; Scheer et al., 2001; Tanigaki et al., 2001; Gaiano and Fishell, 2002). We have shown previously that in the mouse forebrain, Notch 1 promotes radial glial identity during embryogenesis and both dispersed and periventricular astrocyte identities postnatally (Gaiano et al., 2000). Although the promotion of glial fate may seem at odds with Notch maintaining a progenitor state, additional studies have found that in some contexts, glia are progenitors (Doetsch et al., 1999; Malatesta et al., 2000; Fischer and Reh, 2001; Noctor et al., 2001, 
2002; Malatesta et al., 2003). For example, several studies have found that radial glia are progenitors during CNS development (Malatesta et al., 2000; Noctor et al., 2001) and that periventricular astrocytes are stem cells in the adult brain (Doetsch et al., 1999).

We continued to examine the role of Notch1 signaling in the embryonic telencephalon and have found that in addition to promoting radial glial character in vivo, Notch1 activation promotes a proliferative response to FGF2 in vitro. This observation prompted us to consider potential interactions between the Notch and FGF pathways and to examine the role of FGF signaling in vivo. Interestingly, we found that FGR receptor 2 (FGFR2) is expressed in many telencephalic radial glia, and that activation of this receptor promotes radial glial morphology. In addition, the ability of Notch1 to promote proliferative responsiveness in FGF2 correlates with FGFR2 expression in progenitors in vivo. However, we also found that activated FGFR2 (ActFGFR2) was insufficient to promote progenitor proliferation in vitro in the absence of ligand. This work identifies a role for the FGF pathway in radial glial progenitors and suggests that the promotion of radial glial character and progenitor proliferation by FGF signaling are mechanistically separable phenomena.

\section{Materials and Methods}

Retroviral constructs and in vivo infection. The method of retrovirus production has been described previously (Burns et al., 1993; Gaiano et al., 1999). The viral vectors used in this study included murine leukemia virus (MLV)-based genomes and the vesicular stomatitis virus envelope glycoprotein (VSV-G). These vectors included the Xenopus EF1 $\alpha$ enhancer-promoter to drive gene expression (Johnson and Krieg, 1994) and an internal ribosome entry sequence (IRES) to allow dicistronic expression. Human placental alkaline phosphatase (PLAP) and green fluorescent protein (GFP) were used as reporter genes.

Mice were maintained according to protocols approved by the Institutional Animal Care and Use Committee at New York University School of Medicine and Johns Hopkins University School of Medicine. Timed pregnant Swiss Webster mice (provided by the Skirball transgenic facility) or CD-1 mice (Charles River, Wilmington, MA) were used for viral injections, and the morning of the vaginal plug was termed embryonic day 0.5 (E0.5). Virus delivery was performed into the telencephalic ventricle at E9.5 using ultrasound-guided imaging as described previously (Gaiano et al., 1999). The C-promoter binding factor (CBF1)-VP16 cDNAs were obtained from Evelyne Manet (Lyon, France) (Waltzer et al., 1995). The activated form of mouse FGFR2 used contained the C342Y mutation in the third Ig domain that results in ligand-independent homodimerization through aberrant disulfide bond formation (provided by A. Mansukhani, New York University School of Medicine, New York, New York) (Mangasarian et al., 1997). The activated forms of human FGFR1 used contained the N546K or K656E mutations in the kinase domain (provided by Moosa Mohammadi, New York University School of Medicine, New York, New York). The activated form of rat ErbB2 used contained the E664V mutation and is oncogenic (provided by S. Burden, Skirball Institute, New York, NY) (Bargmann et al., 1986). ActFGFR2 and ActErbB2 were cloned into the retroviral vector pCLE (Gaiano et al., 1999) for in vivo infections. CBF1-VP16 and ActFGFR2 were cloned into a retroviral vector called pCEG, which is like pCLE but with GFP in place of PLAP after the IRES sequence.

Cell dissociation, isolation, and in vitro culturing. Virally infected brains were harvested at E12.5 or E14.5. The tissue to be dissociated was minced, washed twice in PBS with $1 \mathrm{~mm}$ EDTA, and incubated in $0.25 \%$ trypsin (Worthington, Freehold, NJ) at $37^{\circ} \mathrm{C}$ for $5 \mathrm{~min}$. Ovomucoid trypsin inhibitor and DNase (both from Worthington) were added, and samples were triturated using a fire-polished Pasteur pipette. Cells were washed twice with DMEM/F12 media, resuspended in PBS, and run through a 40 $\mu \mathrm{m}$ cell strainer (Falcon, Franklin Lakes, NJ) before sorting. Cells expressing GFP were isolated using a Coulter (Beckman Coulter, Inc., Fullerton, CA) Epics Elite Cell Sorter (New York University) or a MoFlo Cell
Sorter (Johns Hopkins University, Baltimore, MD). Where indicated in Figure $2 c$, cells were sorted directly into 96-well dishes. For all other experiments, cells were sorted into $5 \mathrm{ml}$ tubes and then transferred to 6 -well dishes and cultured at clonal density $(<1-2$ cells/ $\mu \mathrm{l})$. We found that the latter collection method generated neurosphere frequencies uniformly lower than the former collection method (see Fig. 2c). This may be the result of cell loss during transfer from $5 \mathrm{ml}$ tubes to 6-well plates. Cells were cultured in DMEM/F12 with the serum supplement B-27 (Invitrogen, San Diego, CA), 2 mm glutamine, and $2 \mu \mathrm{g} / \mathrm{ml}$ heparin. When FGF2 and epidermal growth factor (EGF) (both from Upstate Biotechnology, Waltham, MA) were included in the media, these factors were used at 20 $\mathrm{ng} / \mathrm{ml}$ and $10-20 \mathrm{ng} / \mathrm{ml}$, respectively. Neurospheres were grown for 7-10 $\mathrm{d}$ and were then either differentiated or passaged to make secondary spheres. Scored neurospheres typically ranged from 50 to $150 \mu \mathrm{m}$ in diameter; however, for ActN1-infected cells grown in EGF in particular, those that retained widespread ActN1 expression, were quite small (as few as $10-15$ cells) (see Fig. $2 b$ ). To promote differentiation, neurospheres were cultured for 2 weeks on poly-D-lysine-coated LabTek II chamber slides (Nunc, Rochester, NY) in DMEM/F12 with 5\% FBS.

DiI labeling of radial glia was performed as follows. At E14.5, forebrain hemispheres were dissected and arranged in groups of three, medial side down, on filter inserts in 6-well plates with enough neurosphere media (without growth factors) to keep the explants hydrated but not submerged. DiI was applied to an area of the ventral pia $\sim 1 \mathrm{~mm}$ in diameter using a fine paintbrush. The meninges were left intact in these explants, because this was found in most cases to keep the DiI restricted to the site of application. Explants were incubated for $4 \mathrm{hr}$ at $37^{\circ} \mathrm{C}$ to permit dye diffusion. Those explants with DiI leakage beyond the range of the focal application were discarded. For the remaining explants, excess DiI was removed together with the meninges, and the ventricular zone (VZ)subventricular zone (SVZ) from the ganglionic eminences (GEs) was isolated for dissociation and fluorescence-activated cell sorting (FACS). Throughout this study, samples were compared using a heteroscedastic Student's $t$ test, and the mean \pm SE is given.

In situ hybridization and antibody staining. The $\alpha$-sense probe used for FGFR2 in situ hybridization was 1760 bp in length and extended from PstI to BglII in the mouse cDNA sequence. A sense probe of the same sequence showed no specific labeling. Hybridization was performed as described previously (Schaeren-Wiemers and Gerfin-Moser, 1993).

The following primary antibodies were used: sheep $\alpha$-PLAP (1:200; American Research, Belmont, MA), mouse $\alpha$-TuJ1 (class III $\beta$-tubulin) (1:200 on sections, 1:2000 on neurospheres; Babco, Richmond, CA), rabbit $\alpha$-GFAP (1:2000; Accurate, Westbury, NY), rabbit $\alpha$-synaptophysin (1:300; provided by E. Ziff, New York University School of Medicine, New York, NY), mouse $\alpha$-Rip (supernatant 1:5; Developmental Studies Hybridoma Bank, University of Iowa, Iowa City, IA), mouse $\alpha$-RC2 (ascites 1:150 on sections, 1:400 on dissociated cells; Developmental Studies Hybridoma Bank), rabbit $\alpha$-brain lipid binding protein (BLBP) (1:3000; provided by N. Heintz, Rockefeller University, New York, NY), and rabbit $\alpha$-FGFR2 (1:100 on sections, 1:1000 on dissociated cells; Santa Cruz Biotechnology, Santa Cruz, CA). Secondary antibodies were obtained from Jackson ImmunoResearch (West Grove, PA) and were raised in donkey. TuJ1 was detected using FITC-conjugated goat $\alpha$-mouse $\operatorname{IgG}_{2 \mathrm{a}}$. Standard immunostaining procedures were used. Antibodies were diluted in PBS plus $2 \%$ normal donkey serum plus $0.2 \%$ Triton X-100.

Forebrain-specific deletion of Notch1. The foxg1::CRE mouse line was kindly provided by J. Hebert and S. McConnell (Stanford University, Palo Alto, CA) (Hebert and McConnell, 2000). The primer sequences used in this study are available on request. Crosses to generate embryos homozygous for floxed Notch1 (N1) and heterozygous for foxg1::CRE are described in Results. These animals were initially identified at E12.5 by the substantially reduced size of their GEs. After isolation of the GE for dissociation, the remaining brain tissue was used to genotype the embryo by PCR. PCR analysis of spheres derived from mutants confirmed the deletion of both copies of Notch1, demonstrating that Notch1 was not essential for all neurosphere growth in FGF2. 
a
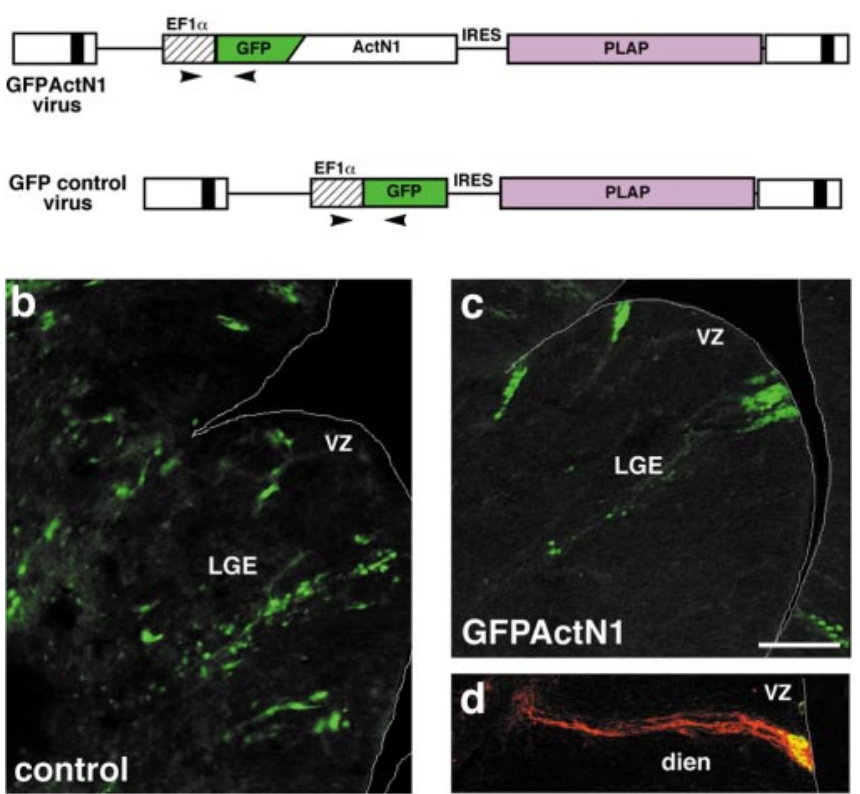

Figure 1. GFP viruses used to identify live infected progenitors. a, Schematics of control and ActN1 viral constructs. The viral backbone for both is p CLE as described previously (Gaiano et al., 1999). Primers used to detect proviral inserts are indicated (arrowheads). $b-d$, Embryos were infected at E9.5 and examined at E14.5. Although control-infected cells were dispersed widely $(b)$, many ActN1-expressing cells were in the ventricular zone $(c, d)$ and possess radial glial morphology as revealed by PLAP expression (d; red). $d$, Diencephalic cells infected with the ActN1-expressing retrovirus show radial glial morphologies similar to those seen in the telencephalon. EF1 $\alpha$, Xenopus EF1 $\alpha$ enhancer-promoter; LGE, lateral ganglionic eminence. Scale bar, $100 \mu \mathrm{m}$. White lines in $b-d$ outline the tissue.

\section{Results}

\section{Notch1 activation enhances neurosphere frequency in FGF2} but not EGF

We infected telencephalic progenitors in utero at E9.5 with retroviruses expressing human PLAP and either a GFP-activated Notch1 fusion protein (referred to herein either as ActN1) or GFP alone (Fig. 1a). The ActN1 protein included GFP at the N terminus fused to a portion of the cytoplasmic tail of Notch1 with constitutive signaling activity (residues 1753-2185). Virus injection was done using ultrasound image guidance and has been described previously (Gaiano et al., 1999).

As we have shown previously (Gaiano et al., 2000), whereas control cells were dispersed throughout both the proliferative and postmitotic zones (Fig. 1b), many ActN1-expressing cells were retained in the $\mathrm{VZ}$ and possessed radial glial characteristics (Fig. $1 c, d$ ). In light of recent studies suggesting that radial glia are progenitors (Malatesta et al., 2000; Noctor et al., 2001, 2002; Malatesta et al., 2003; Anthony et al., 2004), we used the neurosphere assay to test the progenitor character of cells infected with ActN1 in vivo. First, telencephalic cells were infected in utero at E9.5 with ActN1 or control virus. At E14.5, the ventricular and subventricular zones of the GEs were dissected, and GFP-positive cells were isolated using FACS. The VZ-SVZ dissection was used for sample comparison because, whereas control-infected cells were distributed throughout both the proliferative and postmitotic zones, most ActN1-infected cells were in the proliferative zone (Figs. 1, 2a). Based on these distinct distributions, comparison of the entire GE might be expected to show a higher progenitor frequency for ActN1-infected cells. Acute in vitro analysis of control and ActN1-infected cells present in our VZ-SVZ dissec- tions showed that both populations expressed the progenitor marker Nestin at the same frequency $(66.2 \pm 1.4 \%$ for controls, $n=3 ; 68.4 \pm 1.4 \%$ for ActN1, $n=5 ; p=0.32$ over two experiments). This result is consistent with our finding that $65.5 \pm$ $2.2 \%(n=3)$ of the cells in our VZ-SVZ dissections incorporated bromodeoxyuridine after in vivo cumulative labeling. Thus, these dissections allowed us to compare the effects of ActN1 and control viruses in a population of cells enriched for the progenitor pool.

After dissociation, GFP-positive cells were isolated by FACS and grown for 7-10 $\mathrm{d}$ in serum-free media including either FGF2, EGF, both, or neither (Fig. $2 a-c$ ). In media without FGF2 or EGF, no neurospheres were observed, demonstrating that Notch 1 activation could not replace the growth factor requirement of this assay. In media with FGF2 and/or EGF, infected cells did grow into neurospheres as expected. However, $\sim 75 \%$ of the spheres were either heterogeneous with respect to GFP (or ActN1) expression or appeared to be entirely GFP negative. This result suggested that either the neurospheres were not clonal, or retroviral silencing occurred during clonal expansion. Visual inspection of postsort samples confirmed that they were composed of single cells, strongly suggesting that the neurospheres were clonally derived. Furthermore, postsort analysis of the GFPpositive pool indicated that there was very little contamination with GFP-negative cells (3-5\%). In addition, PCR confirmed that the GFP-negative cells in primary neurospheres contained proviral inserts (see below).

When sorted progenitors were plated in media containing FGF2 (with or without EGF), ActN1-expressing progenitors formed spheres 3.7-fold more efficiently than control progenitors (e.g., $22.7 \pm 4.8 \%$ for ActN1; $6.1 \pm 2.0 \%$ for controls; $n=4$ each; $p<0.02$ over four experiments into 96-well dishes with FGF2 alone) (Fig. 2c). In contrast, no significant increase in neurosphere frequency was observed in media containing EGF alone $(2.8 \pm 1.2 \%$ for ActN1; $1.5 \pm 0.8 \%$ for controls; $n=3$ each; $p=$ 0.44 over three experiments). The finding that expression of ActN1 in vivo promoted proliferative responsiveness to FGF2 can be interpreted in the following two ways: (1) Notch1 activation in vivo influences the fate of telencephalic progenitors in a manner that indirectly leads to enhanced proliferative responsiveness to FGF, or (2) Notch1 signaling influences the response to FGF by more directly regulating FGF signaling cascades.

To further examine the effect on Notch1 activation on the responsiveness of telencephalic progenitors to FGF2, we performed in vitro infections of VZ-SVZ cells isolated from the GEs of E14.5 brains with viruses expressing either ActN1 or GFP alone. Cells exposed to virus were incubated overnight to allow proviral integration and expression, and GFP-positive cells were isolated the next day by FACS. Infected cells were plated into neurosphere media containing FGF2 or EGF, and neurosphere frequency was scored 1 week later. Similar to the results above, cells infected in vitro with ActN1 had a 3.2-fold increased neurosphere frequency in FGF2 compared with controls (17.7 $\pm 1.0 \%$ for ActN1; $5.6 \pm 0.8 \%$ for controls; $n=8$ for both from four experiments; $p<0.001$ ) (Fig. $2 d$ ). Also consistent with the in vivo infections, this enhancement was not seen in EGF $(7.4 \pm 0.7 \%$ for ActN1; $6.8 \pm 0.4 \%$ for controls; $n=8$ for both from four experiments; $p=0.47$ ). These data indicate that Notch 1 activation can rapidly influence the growth factor responsiveness of telencephalic progenitors and support the idea that Notch 1 is directly influencing the response of those cells to FGF. 
a

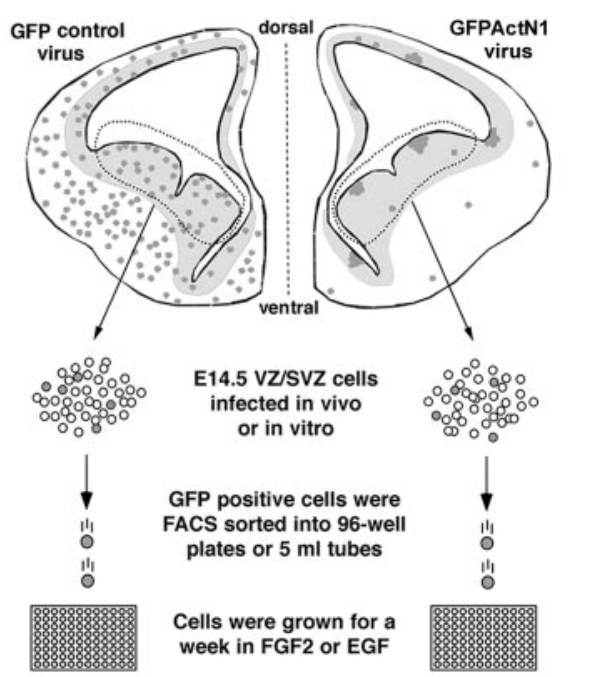

b

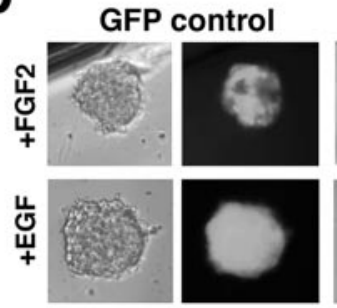

\section{GFPActN1}

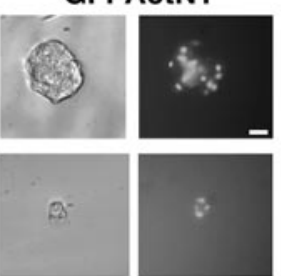

C

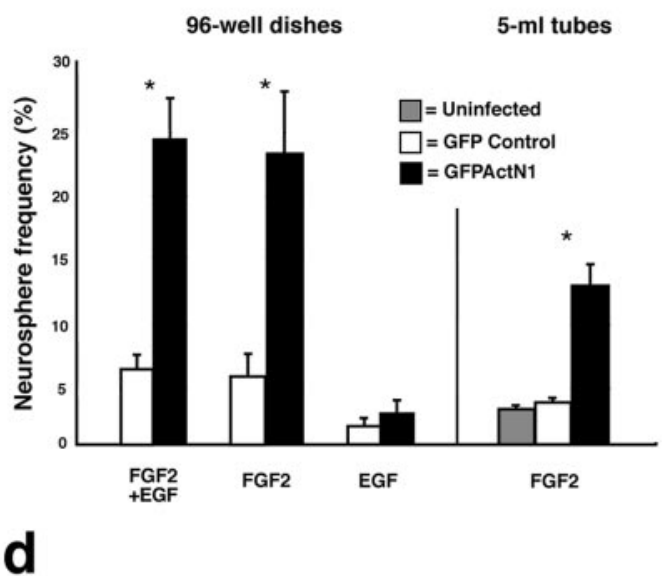

e

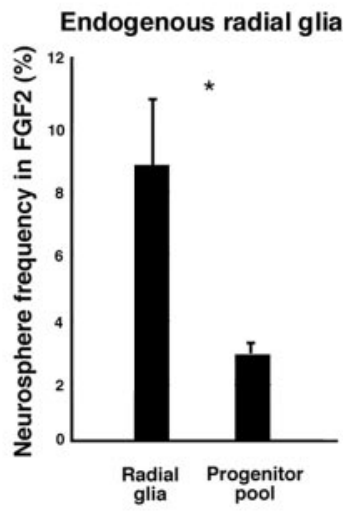

Figure 2. Notch increases the frequency of progenitors that proliferate in FGF2. a, Schematic depiction of approach used to isolate cells expressing either GFP or ActN1. In vivo infections were performed at E9.5, and infected cells were isolated at E14.5 either directly into 96 -well dishes or into $5 \mathrm{ml}$ tubes (see Materials and Methods). $b$, Neurospheres derived from cells infected with control or ActN1 virus in either FGF2 or EGF. In EGF, ActN1 neurospheres were often quite small. Scale bar, $25 \mu \mathrm{m}$. c, In FGF2, ActN1-expressing cells were 3.7-fold more likely to grow into neurospheres than controls. This increase was not seen in EGF. ${ }^{*} p<0.02$. d, Comparison of the effects of ActN1 and CBF1-VP16 after in vitro infection. Cells from E14.5 ganglionic eminence VZ-SVZ dissections were infected in vitro and isolated by FACS the next day. Similar to the results in c, ActN1-infected cells were threefold more likely to grow into neurospheres in FGF2 but not EGF (left). As with ActN1, GE progenitors infected with CBF1-VP16 are threefold more likely to grow into spheres in FGF2 (center). In contrast to ActN1, GE progenitors infected with CBF1-VP16 are threefold more likely to form

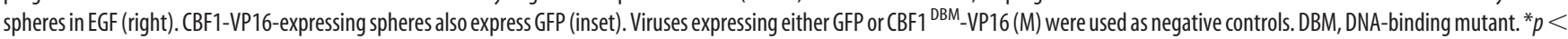
0.02.e, Endogenous GE radial glia isolated using focal Dil application and FACS formed neurospheres efficiently in FGF2. ${ }^{*} p<0.03$.

\section{Activation of CBF1 targets enhances neurosphere frequency in FGF2 and EGF}

The primary effector of Notch signaling is the DNA binding protein CBF1. After ligand stimulation, the Notch intracellular domain translocates to the nucleus and converts CBF1 from a repressor into a transcriptional activator, thus upregulating Notch target genes. In recent years, increasing evidence has supported the idea that Notch receptors can signal in a CBF1-independent manner as well (Martinez Arias et al., 2002). The mechanism of such signaling remains unclear, although interactions with Deltex and the Wnt pathway have been implicated. Therefore, we sought to determine the effect of direct activation of CBF1 targets on neurosphere frequency in FGF2 and EGF. This was achieved by expressing a CBF1-VP16 fusion protein designed to convert CBF1 into a Notch-independent transcriptional activator (Waltzer et al., 1995). As a negative control, we used a CBF1VP16 fusion protein containing a form of CBF1 mutated to abolish DNA binding activity (CBF1 $\left.{ }^{\mathrm{DBM}}-\mathrm{VP} 16\right)$.

To test the effect of activating CBF1 targets, E14.5 ventral telencephalic progenitors were infected in vitro with dicistronic retroviruses expressing CBF1-VP16, CBF $1^{\mathrm{DBM}}-\mathrm{VP} 16$, or PLAP each together with GFP. Infected cells were isolated by FACS and plated into neurosphere media, including FGF2 or EGF, and spheres were scored 1 week later. Consistent with the Notch1 results presented above, expression of CBF1-VP16 promoted a threefold increase in neurosphere frequency in FGF2 compared with expression of $\mathrm{CBF} 1{ }^{\mathrm{DBM}}-\mathrm{VP} 16$ or GFP alone $(16.3 \pm 1.7 \%$ for CBF1-VP16; $5.9 \pm 1.6 \%$ for CBF1 ${ }^{\text {DBM }}-\mathrm{VP} 16$; and $5.5 \pm 0.5 \%$ for GFP; $n=6$ each; $p<0.002$ over three experiments) (Fig. $2 d$ ). This result suggests that Notch 1 increases neurosphere frequency in FGF2 in a CBF1-dependent manner. Interestingly, in contrast to the Notch1 result presented above, CBF1-VP16 promoted a threefold increase in neurosphere frequency in EGF $(5.8 \pm 0.9 \%$ for CBF1-VP16, $n=4 ; 1.8 \pm 0.5 \%$ for CBF1 ${ }^{\text {DBM }_{-}}-\mathrm{VP} 16, n=5$; $1.8 \pm 0.6 \%$ for GFP, $n=3 ; p<0.01$ over three experiments) (Fig. $2 d$ ). This result suggests that the Notch pathway can, under certain circumstances, promote EGF responsiveness.

\section{ActN1-infected progenitors are multipotent and} can self-renew

The observation that ActN1 enhanced the frequency of neurospheres in FGF2 raised the possibility that Notch1 signaling was promoting multipotent progenitor-stem cell character. To test this notion, the developmental potential and self-renewal of ActN1-expressing progenitors was examined. Differentiated primary control and ActN1 neurospheres were immunostained to detect the cell type-specific markers TuJ1 (neurons), synaptophysin (neurons), Rip (oligodendrocytes), and GFAP (astrocytes). The majority (see below) of both ActN1 and control neurospheres (data not shown) expressed markers of multiple cell 


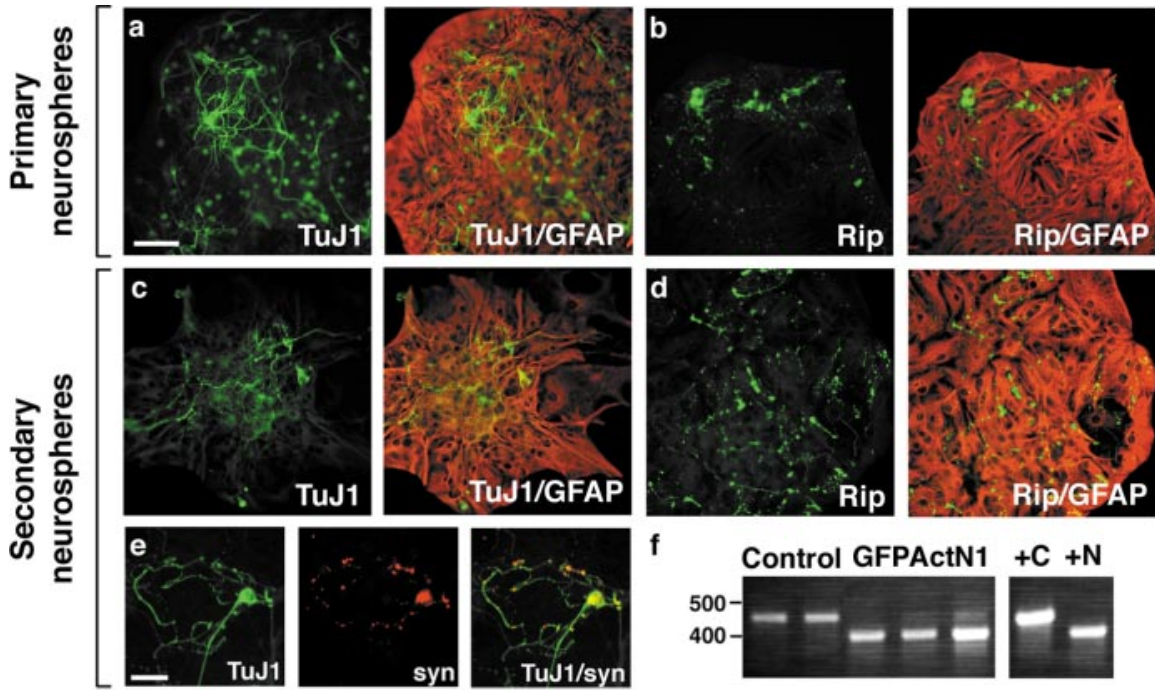

Figure 3. Forebrain cells expressing ActN1 are multipotent and self renew. $a, b$, Primary neurospheres expressed markers of multiple cell types (TuJ1, neurons; Rip, oligodendrocytes; GFAP, astrocytes), indicating that the original cells were multipotent. Continued expression of ActN1 in a was nuclear localized, whereas TuJ1 staining labeled neurites. In many neurospheres, some, if not all, retroviral expression was silenced. $c-e$, Primary spheres were dissociated and grown into multipotent secondary spheres. Neurons in these spheres expressed the synaptic vesicle marker synaptophysin (e).f,PCR confirmed that individual GFP-negative secondary spheres contained proviral inserts and suggested that proviral silencing had occurred. The primers used are depicted in Figure $1 a$ (arrowheads). Positive controls are shown for each ( $C$, control; N, ActN1). Note that the band for control virus is slightly larger because of the presence of additional polylinker sequence between the promoter and GFP. Scale bars: $a-d, 100 \mu \mathrm{m} ; e, 50 \mu \mathrm{m}$.

types, indicating that the initially plated cells were multipotent (Fig. 3a,b). Consistent with work showing that Notch inhibits differentiation of neurons and oligodendrocytes (Nye et al., 1994; Wang et al., 1998), markers for those cells types were only detected in ActN1 spheres that exhibited at least partial retroviral silencing. Nevertheless, the generation of multiple cell types from individual ActN1-expressing cells grown in FGF2 indicated that Notch1 signaling does not block multipotent progenitor character. This result demonstrates that, unlike previous findings in neural crest stem cells (Morrison et al., 2000) and adult hippocampal progenitors (Tanigaki et al., 2001), transient Notch activation does not restrict embryonic forebrain progenitors to a glial fate.

To determine whether ActN1-expressing cells could selfrenew, primary spheres were dissociated and replated at clonal density $(<1-2$ cells/ $\mu \mathrm{l})$ in neurosphere media containing FGF2. As a result of retroviral silencing, both GFP-positive and GFPnegative secondary spheres were obtained from control and ActN1 primary spheres. To confirm the presence of proviral inserts, PCR was performed on individual GFP-negative control and ActN1 secondary spheres (Fig. $3 f$ ). For both viruses, all GFPnegative spheres tested were found to contain proviral inserts $(n=8$ for each). Markers for all three cell types were detected in differentiated secondary spheres (Fig. $3 c-e$ ), demonstrating that the primary sphere cells from which they were derived were multipotent, and that ActN1-expressing cells could self-renew. Thus, telencephalic progenitors maintained by Notch 1 activation can exhibit stem cell character.

\section{Radial glia can form neurospheres in FGF2}

Our observations that Notch1 promotes both radial glial and FGF-responsive progenitor identities in the GEs suggested that some endogenous GE radial glia might be FGF-responsive progenitors. To address this directly, we focally applied the lipophilic dye DiI to the pial surface of the ventral region of E14.5 forebrain hemispheres. Explants were incubated for $4 \mathrm{hr}$ at $37^{\circ} \mathrm{C}$ to permit DiI diffusion, and the GE VZ-SVZ was then dissected away and dissociated. Using this protocol, cells in the VZ-SVZ with long radial processes were found to be labeled with DiI. Similar approaches have been used successfully by others to specifically label radial glia (Voigt, 1989; Malatesta et al., 2000; Noctor et al., 2002). Those cells were isolated using FACS and grown in neurosphere media including FGF2. Dissociated cells from VZ-SVZ dissections of explants without DiI labeling served as controls

Scoring at 1 week revealed that isolated radial glia were threefold more likely to form neurospheres in FGF2 than control cells $(8.8 \pm 2.0 \%$ for isolated radial glia; $3.0 \pm 0.3 \%$ for VZ-SVZ cells; $n=4$ each; $p<0.03$ over four experiments) (Fig. 2e). One potential concern with this approach might be the presence of labeled neurons, because pial application of DiI is likely to label many neurons in proximity to the pial surface. However, such contaminants would reduce, not enhance, the neurosphere frequency. Thus, our data support the idea that at least some radial glia are progenitors that can proliferate into neurospheres in FGF2. This work is also consistent with the idea that Notch1 maintains an FGF responsive progenitor with radial glia morphology.

Deletion of Notch1 decreases neurosphere frequency in FGF2 To investigate further the role of Notch1 signaling in telencephalic progenitors, we used CRE-loxP recombination to delete Notch1 in the telencephalon. Mice homozygous for a "floxed" allele of Notch1 (flanked by loxP sites) (Radtke et al., 1999) were crossed to mice heterozygous for both the floxed Notch1 allele and CRE recombinase knocked into the foxg1 locus (foxg1::CRE) (Hebert and McConnell, 2000). Because foxg1 (formerly called $\mathrm{BF} 1$ ) is expressed throughout the telencephalon from $\sim \mathrm{E} 8$, expression of CRE recombinase from this locus deleted Notch1 in the telencephalon of embryos homozygous for floxed Notch1. At E12.5, embryos heterozygous for foxg1-CRE and homozygous for floxed Notch 1 (called Notch $1^{\mathrm{TEL}}$ mutants hereafter) could be identified morphologically by the reduced size of forebrain structures, in particular the ganglionic eminences (Fig. 4). Subsequent analysis confirmed the genotype of mutant embryos (Fig. $4 g$ ) and that the recombination event to delete Notch1 had occurred as expected (data not shown).

To examine the state of radial glia in mutant forebrains, samples were immunostained for the radial glial markers RC2 and BLBP, both of which were detected in cells with radial morphology (Fig. $4 d, e$ ). This result demonstrates that Notch1 is not essential for the maintenance of all radial glial cell types. Based on the model that most or all VZ cells possess radial glial morphology, this result is consistent with the fact that Notch ${ }^{\text {TEL }}$ mutants do possess a VZ. Regardless, mutants exhibited a significant reduction in the size of the GEs, indicating that progenitor pool output was compromised in the absence of Notch 1 signaling. The expression of Notch2 and Notch3 in the telencephalic VZ (Irvin et 

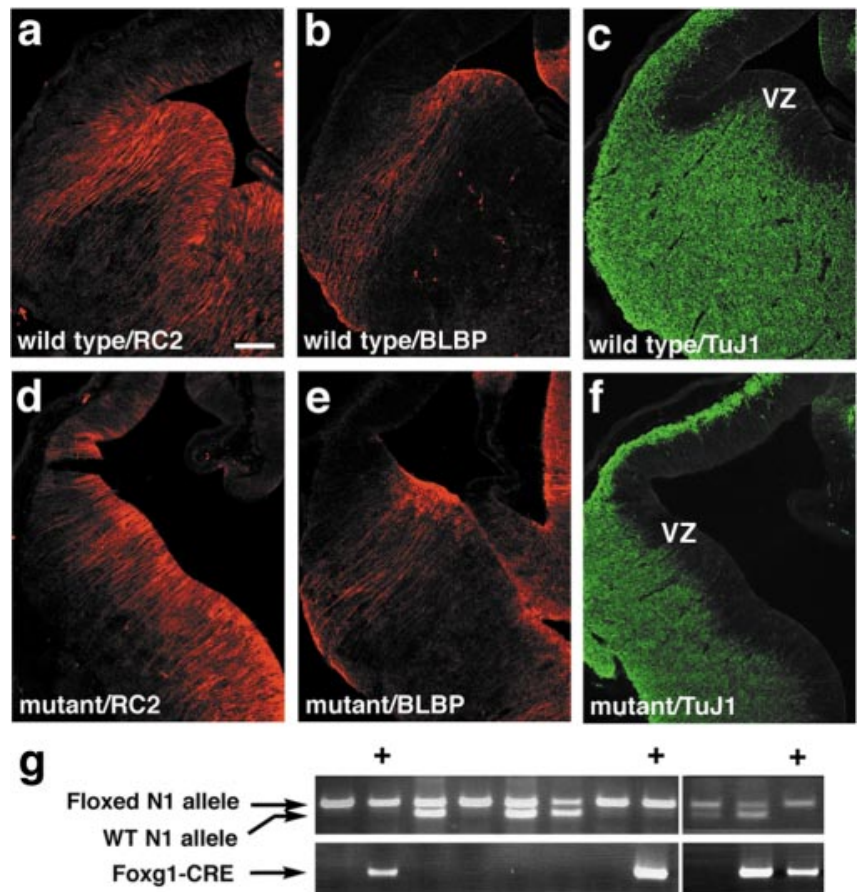

$+$

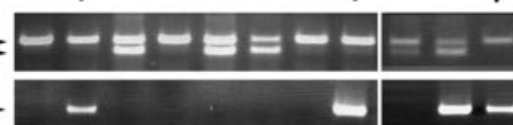

h

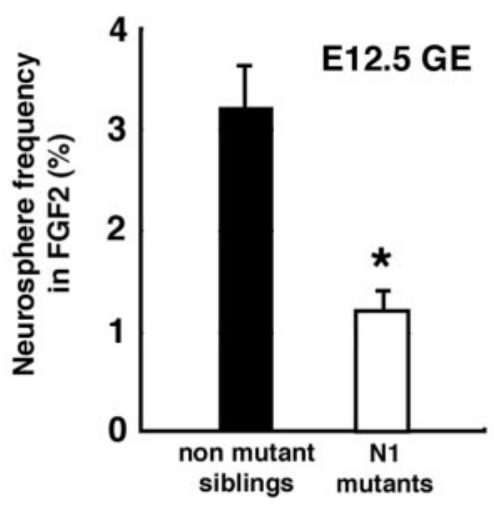

Figure 4. Deletion of Notch1 in the forebrain reduces neurosphere frequency in FGF2. Embryos were generated with a forebrain-specific deletion of Notch 1 (N1). AtE12.5, both wild type $(a-c)$ and mutants $(d, e)$ expressed the radial glial markers $\operatorname{RC}(a, d)$ and BLBP $(b, e)$ as well as the neuronal marker TuJ1 $(c, f)$. However, the size of forebrain structures was substantially reduced in mutants because of a reduction in postmitotic neurons (see TuJ1 staining in c and $f$ ). $g$, Embryos homozygous for floxed N1 and heterozygous for foxg1-CRE were identified by PCR. In the top panel, the larger band indicates the presence of the floxed Notch1 allele, whereas the lower band indicates the presence of the wild-type Notch1 allele. Thus, embryos homozygous for the floxed Notch1 allele lacked the lower band. Mutant embryos (+) were identified as those both homozygous for the floxed Notch1 allele and heterozygous for the Foxg1-Cre. $h$, Neurospheres from wild-type and mutant embryos were generated, and mutant cells showed a 2.7-fold decrease. Scale bar, $100 \mu \mathrm{m} .{ }^{*} p<0.01$.

al., 2001) may provide redundant function, suggesting that deletion of all Notch pathway signaling might have a more severe effect.

Although Notch $1^{\text {TEL }}$ mutants possess radial glia, it remained possible that a subset of the radial glial progenitor pool might be reduced or absent. Thus, we tested the effect of Notch $1^{\mathrm{TEL}}$ deletion on neurosphere frequency in FGF2. GE cells from E12.5 mutant and wild-type embryos were compared, and cells derived from Notch $1{ }^{\text {TEL }}$ mutants were 2.7-fold less likely to form neurospheres in FGF2 than cells derived from wild-type littermates $(1.2 \pm 0.1 \%$ for mutants; $3.2 \pm 0.4 \%$ for controls; $n=7$ each; $p<$ 0.002 over three experiments) (Fig. $4 h$ ). These data are consistent both with our gain-of-function work showing that Notch1 activation increases neurosphere frequency in FGF2 and with the loss-of-function work of others suggesting that Notch signaling is required for neural stem cell maintenance (Ohtsuka et al., 2001; Hitoshi et al., 2002).

\section{Notch1 increases FGF neurosphere frequency in regions expressing FGFR2}

The promotion of neurosphere frequency in FGF2 by Notch1 prompted us to examine FGF signaling in telencephalic progenitors. In particular, we focused on FGFR2 because in situ hybridization and antibody staining revealed that this receptor is expressed in the VZ during development, and like Notch1 (Gaiano et al., 2000) is expressed in radial glia (Fig. $5 a-c$ ). FGFR2 is not expressed in all telencephalic structures, however, and high levels of expression were detected in the neocortex and the ganglionic eminences, whereas little to no expression was evident in the hippocampus and septum (Fig. $5 a-c, e$ ).

The lack of FGFR2 expression in the septum raised the question of would Notch1 activation in septal progenitors enhance neurosphere frequency as it did in the GEs? To examine this issue, we infected the embryonic telencephalon in utero at E9.5 with viruses expressing either ActN1 or GFP. At E14.5, infected GE or septal progenitors were isolated using FACS and plated into neurosphere media, including FGF2. As expected, expression of ActN1 in GE cells resulted in a 3.7-fold increase in neurosphere frequency compared with controls $(13.7 \pm 1.5 \%$ for ActN1, $n=$ 5 ; $3.7 \pm 0.6 \%$ for controls, $n=4$; $p<0.001$ over four experiments) (Fig. $5 d$ ). In contrast, this effect was not seen in septal cells $(0.9 \pm 0.1 \%$ for both ActN1, $n=5$, and controls, $n=4 ; p=0.92$ over four experiments). Consistent with a potential role for FGFR2 in mediating the ability of Notch1 to increase neurosphere frequency in FGF2, ActN1-infected cells did not upregulate FGFR2 expression in the septum (Fig. 5e).

The correlation between FGFR2 expression and the ability of Notch1 to increase FGF neurosphere frequency suggests that signaling through FGFR2 could mediate this effect where FGFR2 is normally expressed. Deletion of FGFR2 will be required to determine whether this is indeed the case. Regardless of which FGFRs are involved, the differential effects of ActN1 in GE and septal progenitors suggests that FGF-responsive progenitors in those structures are maintained using different molecular mechanisms.

Constitutively active FGFR2 promotes radial glial fate in vivo The coincidence of radial glial expression of FGFR2 in regions where Notch 1 activation promoted FGF neurosphere frequency prompted us to consider the effect of FGFR2 activation on progenitors in vivo. To do so, we generated a virus expressing a constitutively active form of FGFR2 (ActFGFR2), which signals via ligand-independent dimerization and receptor tyrosine kinase (RTK) activation, and causes Crouzon Syndrome in humans (Mangasarian et al., 1997). Mouse embryos were infected with ActFGFR2 at E9.5 and analyzed 3-5 d later to determine the effect of continuous FGFR2 signaling. At both E12.5 and E14.5, cells infected with ActFGFR2 virus adopted radial glial morphology and expressed the radial glial marker RC2 (Fig. 6). Acute dissociation of total E14.5 GEs from infected brains showed that cells infected with ActFGFR2 expressed RC2 at a frequency threefold higher than control-infected cells $(57.6 \pm 1.7 \%$ for ActFGFR2, $n=5 ; 18.6 \pm 2.4 \%$ for controls, $n=4 ; p<0.001$ over two experiments) (Fig. 6j,k). The promotion of radial glial morphology and gene expression by ActFGFR2, together with the endogenous expression of FGFR2 in radial glia, suggests that signaling 
a

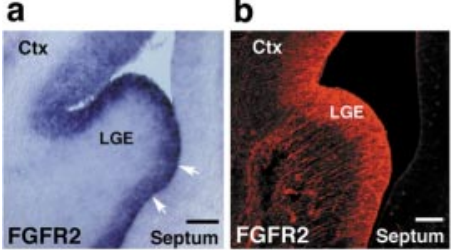

d

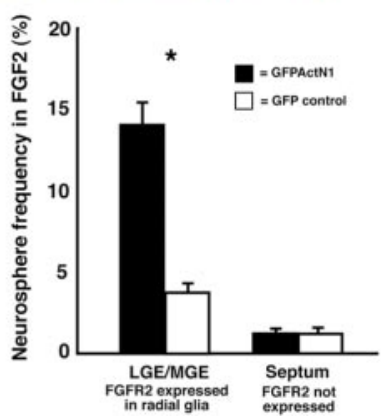

c

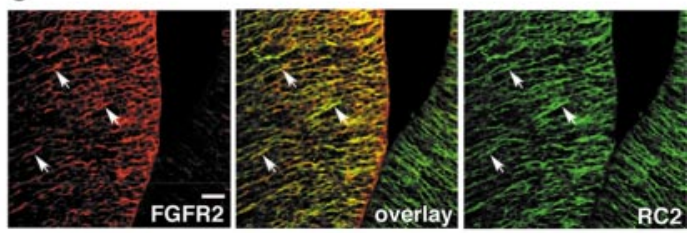

e

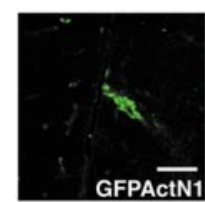

GFPActN
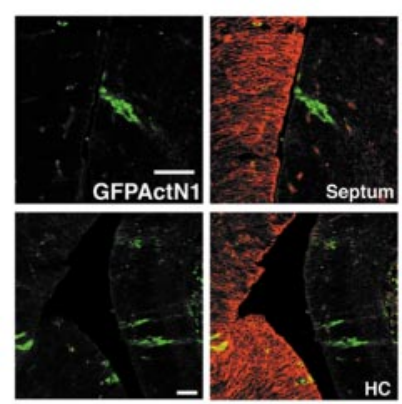

Figure 5. FGFR2 is expressed in radial glia in regions where Notch1 promotes neurosphere frequency in FGF2. $a$, In situ hybridization to detect FGFR2 expression shows expression in the VZ of the cortex (Ctx) and ganglionic eminences [lateral ganglionic eminence (LGE) is shown] but not the septum. The edge of the LGE is indicated (arrows). b, FGFR2 protein was detected throughout the VZ and in radial glial processes in the GEs but not the septum. C, Double labeling showing that FGFR2 is coexpressed with the radial glial marker RC2. $d, \mathrm{GE}$ cells form neurospheres more efficiently in response to ActN1, whereas septum cells do not. ${ }^{*} p<0.02$. e, Cells expressing ActN1 in medial structures such as the septum and hippocampus (HC) do not upregulate FGFR2. Scale bars: $a, b, 100 \mu \mathrm{m} ; c, 25 \mu \mathrm{m} ; e, 50 \mu \mathrm{m}$.
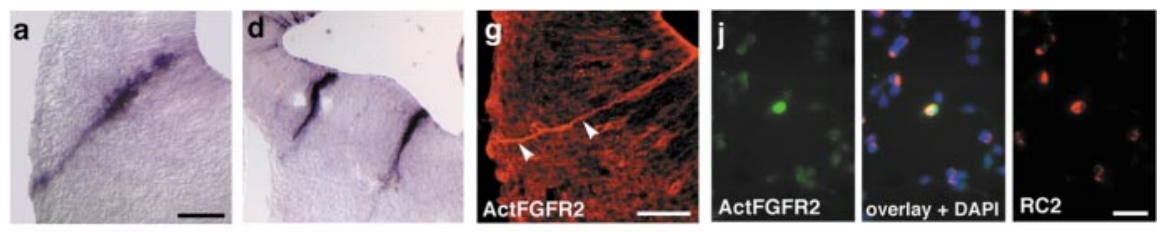

b
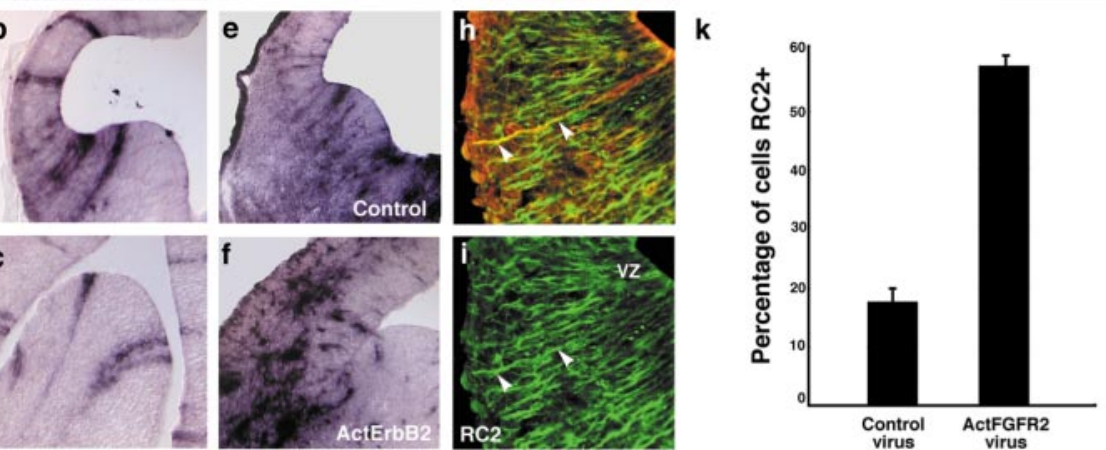

Figure 6. Activated FGFR2 promotes radial glial character in vivo. Infected cells were visualized by histochemical staining for PLAP. $a-d$, Cells expressing ActFGFR2 at E12.5 $(a, b, d)$ and at E14.5 (c) have radial glial morphology. Control-infected cells are more widely dispersed ( $e$; also see Fig. 1b). $f$, Expression of activated ErbB2 does not promote radial glial identity at E14.5, suggesting that this effect is not simply the result of generic RTK activation. $g-i$, ActFGFR2-infected cells express the radial glial marker RC2 in vivo.j, $k$, To quantify RC2 expression, samples were infected at E9.5 with a dicistronic virus expressing ActFGFR2 and GFP or a control GFP virus. $j$, Total GE tissue was dissociated and stained at E14.5 to detect RC2 expression. $k$, GE cells expressing ActFGFR2 were threefold more likely to express RC2 than control-infected cells. Scale bars: (in $a$ ) $a, f, 100 \mu \mathrm{m}$; (in $a$ ) $b-e, 200 \mu \mathrm{m}$; $g, 100 \mu \mathrm{m} ; j, 50 \mu \mathrm{m}$.

(Patten et al., 2003; Schmid et al., 2003). Interestingly, infection of telencephalic progenitors in vivo with activated ErbB2 at E9.5 did not promote radial glial identity, and instead most infected cells migrated to postmitotic areas of the telencephalon (Fig. 6f). It remains possible that different levels of ErbB2 signaling in vivo might have different effects, and that more modest signaling would promote radial glial character. Nevertheless, the observation that ActFGFR2 promotes radial glial character in vivo, whereas ActErbB2 does not, suggests that telencephalic progenitors can respond differently to these two RTK signals.

The observation that ActFGFR2, like ActN1, promoted radial glial identity raised the following question: would FGFR2 signaling in GE progenitors, like ActN1, enhance neurosphere frequency in FGF2? To test this possibility, at E9.5, we injected either control GFP virus or a virus expressing ActFGFR2 and GFP. At E12.5 or E14.5, GFP-positive cells from the VZSVZ of the infected GEs were isolated using FACS and were plated into neurosphere media containing FGF2. At E12.5, ActFGFR2-infected cells did exhibit a modest 1.7 -fold increase in neurosphere frequency compared with controls (6.8 \pm $0.7 \%$ for ActFGFR2, $n=8 ; 4.1 \pm 0.3 \%$ for controls, $n=4 ; p<0.01$ over three experiments) (Fig. 7a). Interestingly, however, by E14.5, this difference was no longer observed, and control and ActFGFR2infected cells had the same neurosphere frequency $(3.6 \pm 1.7 \%$ for ActFGFR2, $n=$ $4 ; 3.7 \pm 0.4 \%$ for controls, $n=5 ; p=0.97$ over four experiments). These data suggested that ligand-independent FGFR2 activation, although sufficient to promote radial glial identity in vivo, was inefficient at enhancing neurosphere frequency, and that additional cues are needed to promote-maintain the neurosphere-forming potential of radial glia. Such cues might be provided by Notch pathway activation, because ActN1 promotes both radial glial character and neurosphere forming potential. Alternatively, the ligand-independent dimerization of FGFR2 ${ }^{\mathrm{C} 342 \mathrm{Y}}$ might not fully recapitulate ligand-induced signaling. through this receptor is involved in the maintenance of a radial glial cell type.

Because FGFR2 is an RTK, and RTKs are known to act through similar signal transduction pathways, we sought to determine whether activation of a different RTK would also promote radial glial identity. In particular, we tested an activated form of the Neuregulin coreceptor ErbB2, because previous work suggested that Neuregulin signaling promotes radial glia identity in vitro (Anton et al., 1997) and that ErbB2 may be a Notch target
Activated FGFRs do not support primary neural progenitor proliferation in vitro

To test the ability of ActFGFR2 to replace the need for FGF ligand in primary telencephalic progenitors, we used the neurosphere assay. Embryos were infected in utero at E9.5 with a virus expressing ActFGFR2 and GFP. At E14.5, ventral telencephalic progenitors were dissociated, and GFP + cells were isolated by FACS and plated into neurosphere media either with or without FGF2. As expected, cells in the presence of FGF2 formed neurospheres at 
a
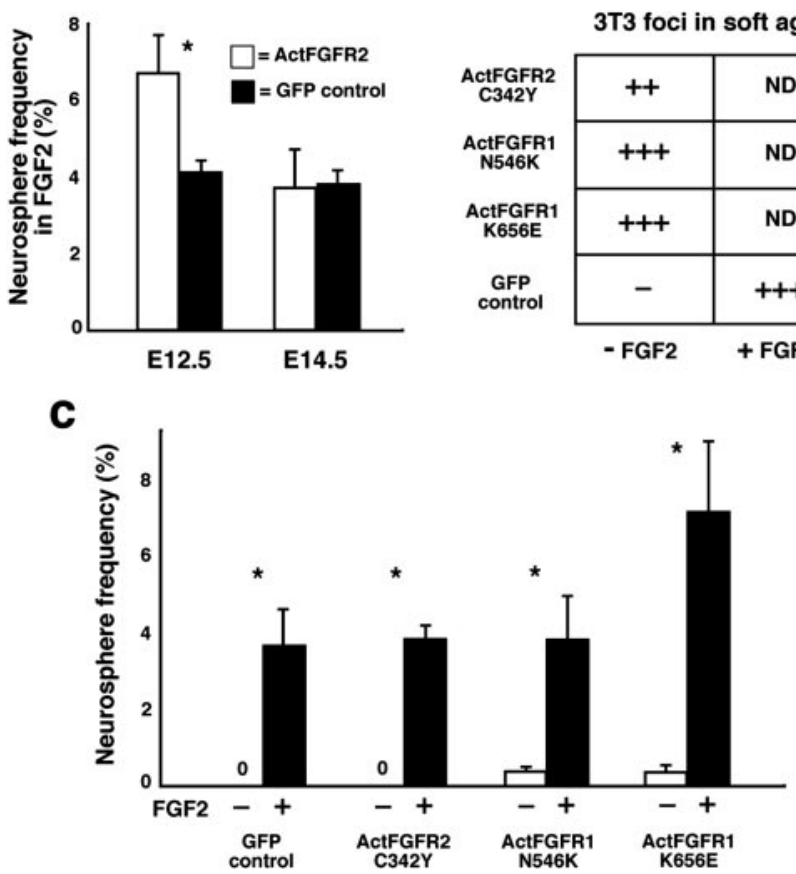

Figure 7. Analysis of the effects of activated FGFRs in telencephalic progenitors in vitro. $a$, Cells were infected in utero at E9.5 with a virus expressing ActFGFR2 and GFP or GFP alone. GFP + cells were isolated by FACS at E12.5 or E14.5 and grown into neurospheres. Although a 1.7-fold increase was observed at E12.5, no effect was evident at E14.5. ${ }^{*} p<0.01$. b, Infected $3 \mathrm{~T} 3$ s were isolated by FACS and plated into media in $0.4 \%$ agar with or without FGF2. Although control-infected cells required FGF2 to proliferate into suspended foci, cells infected with the activated receptors did not. c, Activated forms of FGFR1 and FGFR2 were insufficient to support neurosphere growth from E14.5 ventral neural progenitors in the absence of ligand. These viral stocks were the same as those used in the $3 \mathrm{~T} 3$ assay shown in $b .{ }^{*} p<0.03$. ND, Not done.

frequencies similar to those routinely observed (Fig. 7, compare $a$ and $c$ ). Remarkably, however, in the absence of FGF2, no neurospheres formed (Fig. 7c), suggesting that FGFR2 ${ }^{\mathrm{C} 342 \mathrm{Y}}$ was insufficient to promote proliferation in the absence of ligand. A trivial possible explanation for this result was that widespread retroviral silencing was preventing the expression of FGFR2 ${ }^{\mathrm{C} 342 \mathrm{Y}}$, and that in the absence of ligand, no FGF signal was being generated at all. However, spheres that formed in FGF2 from ActFGFR2-infected cells did not exhibit widespread retroviral silencing, indicating that this explanation was highly unlikely. All told, these data suggested that although FGFR2 ${ }^{\mathrm{C} 342 \mathrm{Y}}$ was sufficient to promote radial glial character in vivo (Fig. 6), it lacked the signaling capacity to promote proliferation in vitro.

There were several possible explanations for the inability of FGFR2 ${ }^{\mathrm{C} 342 \mathrm{Y}}$ to support neurosphere formation in the absence of ligand. First, something specific to the C342Y mutation might limit the activation of downstream signaling cascades. Alternatively, activated forms of FGFR1, rather than FGFR2, might relieve the need for ligand in the neurosphere assay, because previous studies have found that FGFR1 is essential for the proliferative response of forebrain progenitors to FGF2 (Tropepe et al., 1999; Ohkubo et al., 2004). In an effort to address both of these concerns, we tested the ability of two activated forms of FGFR1 to support neurosphere formation in the absence of FGF2. The forms used contained the kinase domain mutations N546K or K656E and were both originally identified as activating mutations in FGFR3 that cause skeletal dysplasia in humans (Robertson et al., 2000). Consistent with our FGFR2 ${ }^{\mathrm{C} 342 \mathrm{Y}}$ results, neither FGFR1 ${ }^{\text {N546K }}$ nor FGFR1 ${ }^{\mathrm{K} 656 \mathrm{E}}$ were able to relieve the need for ligand during neurosphere formation (Fig. 7c). The ability of the activated forms of FGFR 1 and 2 encoded by our viral stocks to promote proliferation was confirmed functionally using the 3 T3 soft agar assay (Fig. 7b). Together, these data suggest that FGF ligands play a role beyond simple receptor activation in promoting primary telencephalic progenitor proliferation.

\section{Discussion}

Notch1 promotes radial glial character and proliferative responsiveness to FGF2

We have shown previously that forebrain progenitors expressing a constitutively active form of Notch 1 become radial glia (Gaiano et al., 2000). Simultaneously, others found that Notch signaling in the retina led to Muller glial fate (Furukawa et al., 2000) and in the neural crest to Schwann cell fate (Morrison et al., 2000). In addition, Notch signaling in adult hippocampal progenitors has been found to promote astrocytic fate (Gaiano et al., 2000; Chambers et al., 2001; Tanigaki et al., 2001). The simplest interpretation of this work was that Notch promoted glial fate. As such, these studies challenged the view that in the vertebrate nervous system, Notch inhibits differentiation and promotes progenitor maintenance.

Contemporary with this work, however, numerous reports have suggested that glial cell types and in particular radial glia can possess progenitor characteristics. For example, radial glia have been observed proliferating and giving rise to neurons in slice culture (Noctor et al., 2001). Other work has shown that shortterm cultures of isolated radial glia can generate neurons and glia in vitro (Malatesta et al., 2000). In addition, fate-mapping work has shown that radial glia can give rise to neurons in vivo (Malatesta et al., 2003; Anthony et al., 2004). Here, we found that radial glia can form neurospheres, further supporting the idea that radial glia are progenitors. These observations, together with our findings that Notch1 promotes radial glial character in vivo and multipotent neurosphere formation in FGF2 in vitro, suggest that radial glia maintained by Notch1 are multipotent progenitors that proliferate in response to FGF.

How does Notch1 signaling maintain proliferative responsiveness to FGF? The promotion of radial glial fate by ActN1 in vivo indicates that Notch 1 is influencing progenitor fate and might be modulating growth factor responsiveness only indirectly. However, the ability of Notch 1 activation in vitro to rapidly increase the fraction of progenitors that proliferate in FGF suggests that Notch may directly influence FGF signaling cascades. How direct that interaction is remains to be determined, although the fact that $\mathrm{CBF} 1$ can also increase neurosphere frequency in FGF2 suggests that Notch1 signaling influences FGF responsiveness by regulating gene expression. In support of our findings that the Notch and FGF signaling pathways interact in telencephalic progenitors, others have shown that FGF ligands can inhibit neuronal differentiation in vitro, but that this effect is dependent on Notch (Faux et al., 2001).

\section{Telencephalic specific deletion of Notch1}

To complement our gain-of-function studies, we examined the effect of deleting Notch 1 from the embryonic telencephalon. Although numerous studies have considered the effect of deleting Notch pathway components on neural development, early lethality has limited these analyses (Ishibashi et al., 1995; Oka et al., 1995; de la Pompa et al., 1997; Hitoshi et al., 2002). By deleting Notch1 specifically in the telencephalon, we were able to circumvent early lethality (standard Notch1 mutants are growth re- 
tarded and die by E11) and to consider the effects of Notch1 deletion on telencephalic progenitors both in vivo and in vitro later in development.

At E12.5 Notch $1{ }^{\text {TEL }}$ mutant embryos contained cells with radial glial morphology and marker expression showing that loss of Notch1 activity was not essential for all radial glia. In light of recent evidence that most or all VZ cells may be radial glia (Noctor et al., 2002), this result is not entirely surprising, because Notch $1{ }^{\text {TEL }}$ mutants still possess a VZ. Nevertheless, mutant animals did show a reduction in the size of forebrain structures, suggesting that the progenitor pool had diminished capacity to produce postmitotic cell types. In addition, mutant brains had a 2.7-fold lower FGF neurosphere frequency than controls supporting the view that Notch1 maintains a progenitor state.

These data suggest that Notch1 is essential for the maintenance of most but not all FGF-responsive progenitors in the GEs. Because Notch 2 and Notch3 are also expressed in forebrain progenitors (Irvin et al., 2001), they may compensate for the loss of Notch1. Alternatively, it remains possible that some FGFresponsive progenitors in the GEs are maintained independent of Notch signaling. Such cells might be akin to those FGFresponsive progenitors present in the septum, which do not appear to be influenced by Notch1 activation.

\section{FGFR signaling in forebrain progenitors}

Neural progenitors that proliferate in FGF2 appear during brain development as early as E8.5 and are present through adulthood. Previous work has found that FGFR1 is essential for the specification of these cells before neurogenesis (Tropepe et al., 1999). In addition to FGFR1, both FGFR2 and FGFR3 are expressed in the telencephalic VZ throughout neurogenesis (Bansal et al., 2003). An interesting distinction between these receptors, however, is that whereas FGFR1 and FGFR3 are expressed widely throughout the telencephalic VZ, FGFR2 expression is weak to absent in medial structures like the septum and hippocampus (Fig. 5). This differential expression of FGFRs, and our finding that Notch1 activation in septal progenitors does not enhance FGF2 neurosphere frequency, suggests that Notch1 may modulate signaling through FGFR2 in regions where FGFR2 is expressed (i.e., the ganglionic eminences). The early lethality of FGFR2 mutants means that a direct test of the role of FGFR2 in forebrain progenitors will require conditional deletion.

To further explore the role of FGF signaling in the telencephalon, we examined the phenotype of forebrain progenitors expressing activated FGFR2 in vivo. Similar to previous results with ActN1, these cells acquired radial glial morphology and expressed the radial glial marker RC2. This result and the fact that FGFR2 is expressed in radial glia both support a role for FGF signaling in radial glial maintenance. Considered together with our findings that Notch signaling can influence the FGF responsiveness of telencephalic progenitors, and the growing literature suggesting that radial glia are progenitors, we believe that Notch and FGF interact to maintain at least a portion of the progenitor pool as proliferating radial glia.

Additional study will be needed to determine which downstream components of the Notch and FGF signaling pathways function and interact to regulate telencephalic progenitor character and proliferation. As a first step toward this goal, we found that the CBF1-dependent component of the Notch signaling cascade is likely to play a role. In addition, we found that although activated FGFR2 has a strong effect on cell fate, it cannot replace the need for ligand in promoting primary neural progenitor proliferation. This latter finding suggests that FGF ligands perform a function more complex than simply activating FGFRs. This idea is consistent with the previous work of others examining the role of FGF ligands in promoting proliferation (Wiedlocha et al., 1994, 1996) and suggests that it may be possible to mechanistically separate different aspects of FGF signaling in neural progenitors.

\section{References}

Anthony TE, Klein C, Fishell G, Heintz N (2004) Radial glia serve as neuronal progenitors in all regions of the central nervous system. Neuron 41:881-890.

Anton ES, Marchionni MA, Lee KF, Rakic P (1997) Role of GGF/neuregulin signaling in interactions between migrating neurons and radial glia in the developing cerebral cortex. Development 124:3501-3510.

Bansal R, Lakhina V, Remedios R, Tole S (2003) Expression of FGF receptors 1, 2, 3 in the embryonic and postnatal mouse brain compared with Pdgfralpha, Olig2 and Plp/dm20: implications for oligodendrocyte development. Dev Neurosci 25:83-95.

Bargmann CI, Hung MC, Weinberg RA (1986) Multiple independent activations of the neuroncogene by a point mutation altering the transmembrane domain of p185. Cell 45:649-657.

Burns JC, Friedmann T, Driever W, Burrascano M, Yee JK (1993) Vesicular stomatitis virus $\mathrm{G}$ glycoprotein pseudotyped retroviral vectors: concentration to very high titer and efficient gene transfer into mammalian and nonmammalian cells. Proc Natl Acad Sci USA 90:8033-8037.

Chambers CB, Peng Y, Nguyen H, Gaiano N, Fishell G, Nye JS (2001) Spatiotemporal selectivity of response to Notch 1 signals in mammalian forebrain precursors. Development 128:689-702.

Chitnis AB (1995) The role of Notch in lateral inhibition and cell fate specification. Mol Cell Neurosci [Erratum (1995) 6(6):311-321] 6(4):311-321.

de la Pompa JL, Wakeham A, Correia KM, Samper E, Brown S, Aguilera RJ, Nakano T, Honjo T, Mak TW, Rossant J, Conlon RA (1997) Conservation of the Notch signalling pathway in mammalian neurogenesis. Development 124:1139-1148.

Doetsch F, Caille I, Lim DA, Garcia-Verdugo JM, Alvarez-Buylla A (1999) Subventricular zone astrocytes are neural stem cells in the adult mammalian brain. Cell 97:703-716.

Faux CH, Turnley AM, Epa R, Cappai R, Bartlett PF (2001) Interactions between fibroblast growth factors and Notch regulate neuronal differentiation. J Neurosci 21:5587-5596.

Fischer AJ, Reh TA (2001) Muller glia are a potential source of neural regeneration in the postnatal chicken retina. Nat Neurosci 4:247-252.

Furukawa T, Mukherjee S, Bao ZZ, Morrow EM, Cepko CL (2000) rax, Hes1, and notch1 promote the formation of Muller glia by postnatal retinal progenitor cells. Neuron 26:383-394.

Gaiano N, Fishell G (2002) The role of notch in promoting glial and neural stem cell fates. Annu Rev Neurosci 25:471-490.

Gaiano N, Kohtz JD, Turnbull DH, Fishell G (1999) A method for rapid gain-of-function studies in the mouse embryonic nervous system. Nat Neurosci 2:812-819.

Gaiano N, Nye JS, Fishell G (2000) Radial glial identity is promoted by Notch1 signaling in the murine forebrain. Neuron 26:395-404.

Hebert JM, McConnell SK (2000) Targeting of cre to the Foxg1 (BF-1) locus mediates loxP recombination in the telencephalon and other developing head structures. Dev Biol 222:296-306.

Henrique D, Hirsinger E, Adam J, Le Roux I, Pourquie O, Ish-Horowicz D, Lewis J (1997) Maintenance of neuroepithelial progenitor cells by DeltaNotch signalling in the embryonic chick retina. Curr Biol 7:661-670.

Hitoshi S, Alexson T, Tropepe V, Donoviel D, Elia AJ, Nye JS, Conlon RA, Mak TW, Bernstein A, van der Kooy D (2002) Notch pathway molecules are essential for the maintenance, but not the generation, of mammalian neural stem cells. Genes Dev 16:846-858.

Irvin DK, Zurcher SD, Nguyen T, Weinmaster G, Kornblum HI (2001) Expression patterns of Notch1, Notch2, and Notch3 suggest multiple functional roles for the Notch-DSL signaling system during brain development. J Comp Neurol 436:167-181.

Ishibashi M, Ang SL, Shiota K, Nakanishi S, Kageyama R, Guillemot F (1995) Targeted disruption of mammalian hairy and Enhancer of split homolog-1 (HES-1) leads to up-regulation of neural helix-loop-helix factors, premature neurogenesis, and severe neural tube defects. Genes Dev 9:3136-3148.

Johnson AD, Krieg PA (1994) pXeX, a vector for efficient expression of cloned sequences in Xenopus embryos. Gene 147:223-226.

Lardelli M, Williams R, Mitsiadis T, Lendahl U (1996) Expression of the 
Notch 3 intracellular domain in mouse central nervous system progenitor cells is lethal and leads to disturbed neural tube development. Mech Dev 59:177-190.

Lewis J (1996) Neurogenic genes and vertebrate neurogenesis. Curr Opin Neurobiol 6:3-10.

Malatesta P, Hartfuss E, Gotz M (2000) Isolation of radial glial cells by fluorescent-activated cell sorting reveals a neuronal lineage. Development 127:5253-5263.

Malatesta P, Hack MA, Hartfuss E, Kettenmann H, Klinkert W, Kirchhoff F, Gotz M (2003) Neuronal or glial progeny: regional differences in radial glia fate. Neuron 37:751-764.

Mangasarian K, Li Y, Mansukhani A, Basilico C (1997) Mutation associated with Crouzon syndrome causes ligand-independent dimerization and activation of FGF receptor-2. J Cell Physiol 172:117-125.

Martinez Arias A, Zecchini V, Brennan K (2002) CSL-independent Notch signalling: a checkpoint in cell fate decisions during development? Curr Opin Genet Dev 12:524-533.

Morrison SJ, Perez SE, Qiao Z, Verdi JM, Hicks C, Weinmaster G, Anderson DJ (2000) Transient Notch activation initiates an irreversible switch from neurogenesis to gliogenesis by neural crest stem cells. Cell 101:499-510.

Noctor SC, Flint AC, Weissman TA, Dammerman RS, Kriegstein AR (2001) Neurons derived from radial glial cells establish radial units in neocortex. Nature 409:714-720.

Noctor SC, Flint AC, Weissman TA, Wong WS, Clinton BK, Kriegstein AR (2002) Dividing precursor cells of the embryonic cortical ventricular zone have morphological and molecular characteristics of radial glia. J Neurosci 22:3161-3173.

Nye JS, Kopan R, Axel R (1994) An activated Notch suppresses neurogenesis and myogenesis but not gliogenesis in mammalian cells. Development 120:2421-2430.

Ohkubo Y, Uchida AO, Shin D, Partanen J, Vaccarino FM (2004) Fibroblast growth factor receptor 1 is required for the proliferation of hippocampal progenitor cells and for hippocampal growth in mouse. J Neurosci 24:6057-6069.

Ohtsuka T, Sakamoto M, Guillemot F, Kageyama R (2001) Roles of the basic helix-loop-helix genes Hes1 and Hes5 in expansion of neural stem cells of the developing brain. J Biol Chem 276:30467-30474.

Oka C, Nakano T, Wakeham A, de la Pompa JL, Mori C, Sakai T, Okazaki S, Kawaichi M, Shiota K, Mak TW, Honjo T (1995) Disruption of the mouse RBP-J kappa gene results in early embryonic death. Development 121:3291-3301.
Patten BA, Peyrin JM, Weinmaster G, Corfas G (2003) Sequential signaling through Notch 1 and erbB receptors mediates radial glia differentiation. J Neurosci 23:6132-6140.

Radtke F, Wilson A, Stark G, Bauer M, van Meerwijk J, MacDonald HR, Aguet M (1999) Deficient $T$ cell fate specification in mice with an induced inactivation of Notch1. Immunity 10:547-558.

Robertson SC, Tynan J, Donoghue DJ (2000) RTK mutations and human syndromes: when good receptors turn bad. Trends Genet 16:368.

Schaeren-Wiemers N, Gerfin-Moser A (1993) A single protocol to detect transcripts of various types and expression levels in neural tissue and cultured cells: in situ hybridization using digoxigenin-labelled cRNA probes. Histochemistry 100:431-440.

Scheer N, Groth A, Hans S, Campos-Ortega JA (2001) An instructive function for Notch in promoting gliogenesis in the zebrafish retina. Development 128:1099-1107.

Schmid RS, McGrath B, Berechid BE, Boyles B, Marchionni M, Sestan N, Anton ES (2003) Neuregulin 1-erbB2 signaling is required for the establishment of radial glia and their transformation into astrocytes in cerebral cortex. Proc Natl Acad Sci USA 100:4251-4256.

Tanigaki K, Nogaki F, Takahashi J, Tashiro K, Kurooka H, Honjo T (2001) Notch1 and Notch3 instructively restrict bFGF-responsive multipotent neural progenitor cells to an astroglial fate. Neuron 29:45-55.

Tropepe V, Sibilia M, Ciruna BG, Rossant J, Wagner EF, van der Kooy D (1999) Distinct neural stem cells proliferate in response to EGF and FGF in the developing mouse telencephalon. Dev Biol 208:166-188.

Voigt T (1989) Development of glial cells in the cerebral wall of ferrets: direct tracing of their transformation from radial glia into astrocytes. J Comp Neurol 289:74-88.

Waltzer L, Bourillot PY, Sergeant A, Manet E (1995) RBP-J kappa repression activity is mediated by a co-repressor and antagonized by the Epstein-Barr virus transcription factor EBNA2. Nucleic Acids Res 23:4939-4945.

Wang S, Sdrulla AD, diSibio G, Bush G, Nofziger D, Hicks C, Weinmaster G, Barres BA (1998) Notch receptor activation inhibits oligodendrocyte differentiation. Neuron 21:63-75.

Wiedlocha A, Falnes PO, Madshus IH, Sandvig K, Olsnes S (1994) Dual mode of signal transduction by externally added acidic fibroblast growth factor. Cell 76:1039-1051.

Wiedlocha A, Falnes PO, Rapak A, Munoz R, Klingenberg O, Olsnes S (1996) Stimulation of proliferation of a human osteosarcoma cell line by exogenous acidic fibroblast growth factor requires both activation of receptor tyrosine kinase and growth factor internalization. Mol Cell Biol 16:270-280. 